\title{
O petróleo e sua destilação: uma abordagem experimental no Ensino Médio utilizando mapas conceituais.
}

\section{Crude oil and its' distillation: an experimental approach in High School using conceptual maps.}

\author{
Nelci Reis Sales de Araújoㅜㄹ Eliana Ap. Silicz Bueno²; Flaveli Ap. de Souza Almeida²; Dionísio Borsato ${ }^{2}$
}

\section{Resumo}

\begin{abstract}
Os mapas conceituais consistem em representações de conceitos organizados em forma de diagrama bidimensional. Neste trabalho, foi explorado o tema "destilação fracionada do petróleo", e os mapas conceituais foram elaborados em períodos pré e pós-atividades, por 43 alunos da $1^{\mathrm{a}}$ e $3^{\mathrm{a}}$ séries do Ensino Médio de uma escola pública de Londrina-PR. O estudo conduziu-se dentro de um contexto de teoria e prática, com implicações no dia a dia de nossa sociedade. O uso do tema motivador e do texto de abertura, como organizadores prévios, permitiu uma ponte cognitiva entre os conhecimentos dos alunos e os novos conceitos. Verificaram-se diferenças entre os mapas elaborados antes e após as atividades, bem como entre os grupos de trabalho. Os alunos estimulados com a técnica elaboraram mapas mais estruturados.
\end{abstract}

Palavras- chave: Mapa conceitual. Petróleo. Tema motivador.

\begin{abstract}
Conceptual maps are representations of ideas organized in the form of bidimensional diagrams. In the present work the theme of oil fractional distillation was explored, and the conceptual maps were elaborated both before and after the activities by 43 students from the $1^{\text {st }}$ and $3^{\text {rd }}$ High School grades of a public school in Londrina - PR. The study was conducted theoretically and in practice, with a daily life approach. The use of the motivational theme and the opening text as previous organizers, enabled the establishment of a cognitive link between the students' previous knowledge and the new concepts. Differences between the maps were verified before and after the activities as well as among the work groups. The students, stimulated by the technique, created better structured maps.
\end{abstract}

Key words: Conceptual map. Crude oil. Motivating theme

\footnotetext{
${ }^{1}$ Colégio Estadual José Aloísio Aragão (Colégio de Aplicação da UEL).

${ }^{2}$ Docente da Universidade Estadual de Londrina, Centro de Ciências Exatas, Departamento de Química.Campus Universitário.

E- mail: dborsato@uel.br
} 


\section{Introdução}

No decurso da história, os métodos de ensino e os processos de aprendizagem foram mudando rapidamente, acompanhando o processo de construção do conhecimento humano. Vários foram os métodos e conceitos empregados como sendo os únicos corretos e aceitos por uma categoria ou classe social. Esses métodos que privilegiaram classes e excluíram outras, formaram opiniões e, quando mal elaborados, destruíram a capacidade de criação do indivíduo.

O aprendizado de Química pelos alunos do Ensino Médio, implica a compreensão das transformações químicas que ocorrem no mundo físico de forma abrangente e integrada. Esse aprendizado deve possibilitar ao aluno a compreensão tanto dos processos químicos, quanto da construção de um conhecimento científico em estreita relação com as aplicações tecnológicas e suas implicações ambientais, sociais, políticas e econômicas. Essa compreensão ajuda o estudante e o professor a terem a necessária visão crítica da ciência (MENEZES, 2001).

Segundo o mesmo autor, o ensino de Química tem-se reduzido à transmissão de informações e leis isoladas, sem qualquer relação com a vida do aluno, dos quais quase sempre se exige memorização, restrita a baixos níveis cognitivos. Enfatiza-se muito a classificação de reações químicas, de soluções, de ácidos, dentre outros, e isso não representa aprendizagens significativas. Reduz-se o conhecimento químico à fórmulas matemáticas e à aplicação de 'regrinhas', que são exaustivamente treinadas, levando à mecanização e não ao entendimento de uma situação-problema. Em outros momentos, o ensino atual privilegia aspectos teóricos, em níveis de abstração inadequados aos dos estudantes.

Os conteúdos na área de Química podem ser trabalhados a partir de temas que permitam a contextualização do conhecimento. Para complementação dos temas, pode-se utilizar a experimentação, no Ensino Médio, com a função pedagógica. Segundo Menezes (2001), a experimentação formal em laboratórios didáticos, por si só, não soluciona o problema de ensino aprendizagem em Química. As atividades experimentais podem ser realizadas na sala de aula por demonstração, em visitas e por outras modalidades. Qualquer que seja a atividade a ser desenvolvida, deve-se ter clara a necessidade de períodos pré e pós atividade, visando à construção dos conceitos. Dessa forma, não se desvinculam 'teoria' e 'laboratório'.

Uma preocupação constante dos educadores na atualidade é a priorização de metodologias aptas a tornar o processo ensino - aprendizado mais produtivo. Heron e Nurrenbern (1999) sugerem a aplicação das metodologias de ensino que seguem a linha construtivista.

Nas últimas décadas houve um crescimento expressivo de pesquisa para o desenvolvimento de instrumentos, técnicas, metodologias ou processos no ensino de Química, notadamente no Ensino Médio, tanto em sala de aula, de campo, como em aulas experimentais. No entanto, as aulas experimentais mostram-se problemáticas, tanto pela ausência de materiais, em laboratórios precariamente equipados, como também continuam sendo vistas sem interesse e motivação por parte dos alunos. Evidencia-se, muitas vezes, a falta de contextualização nestas aulas, que envolvem o ensino - aprendizagem de Química (BENETASSO et al., 2002).

Quando incorporadas na instrução de laboratório, as técnicas modernas de ensino - aprendizagem, divulgadas por estudos publicados com diferentes enfoques, quando incorporadas na instrução de laboratório, ajudam a melhorar a compreensão dos conceitos relacionados dos alunos. Técnicas gráficas como a de mapa conceitual e diagrama em "Vê", estão baseadas em uma perspectiva construtivista. Tais estratégias instrutivas possuem contribuições efetivas para a aprendizagem significativa, servindo para avaliar o progresso do estudante (NOVAK; GOWIN, 1996). 
O procedimento gráfico "Mapa Conceitual” é uma técnica instrutiva que ajuda os estudantes a guiar e aplicar conceitos químicos nas atividades de laboratório, integrando-os com a realidade do cotidiano. Esta técnica pode ser usada para impor suas próprias estruturas conceituais, através da construção, reconstrução e inter-relações de conceitos de Química, tendo como base um tema organizador (MOREIRA; NOVAK, 1988).

O trabalho enfoca a exploração de um tema organizador, relacionado ao cotidiano dos alunos, e lança-se mão da técnica de mapas conceituais para investigar nos alunos a construção, reconstrução e as inter-relações de conceitos da Química numa aprendizagem significativa, bem como relacioná-los com o seu cotidiano, para sua completa formação e cidadania.

\section{Materiais e Métodos.}

\section{Petróleo}

Foi utilizado, no procedimento de destilação, o petróleo árabe leve, cedido pelo Laboratório de Fluorescência e Ressonância Paramagnética Eletrônica da Universidade Estadual de Londrina.

\section{Destilação.}

Para demonstrar o processo de destilação, foi utilizado o equipamento mostrado na Figura1.

Destilador - Destilação Fracionada

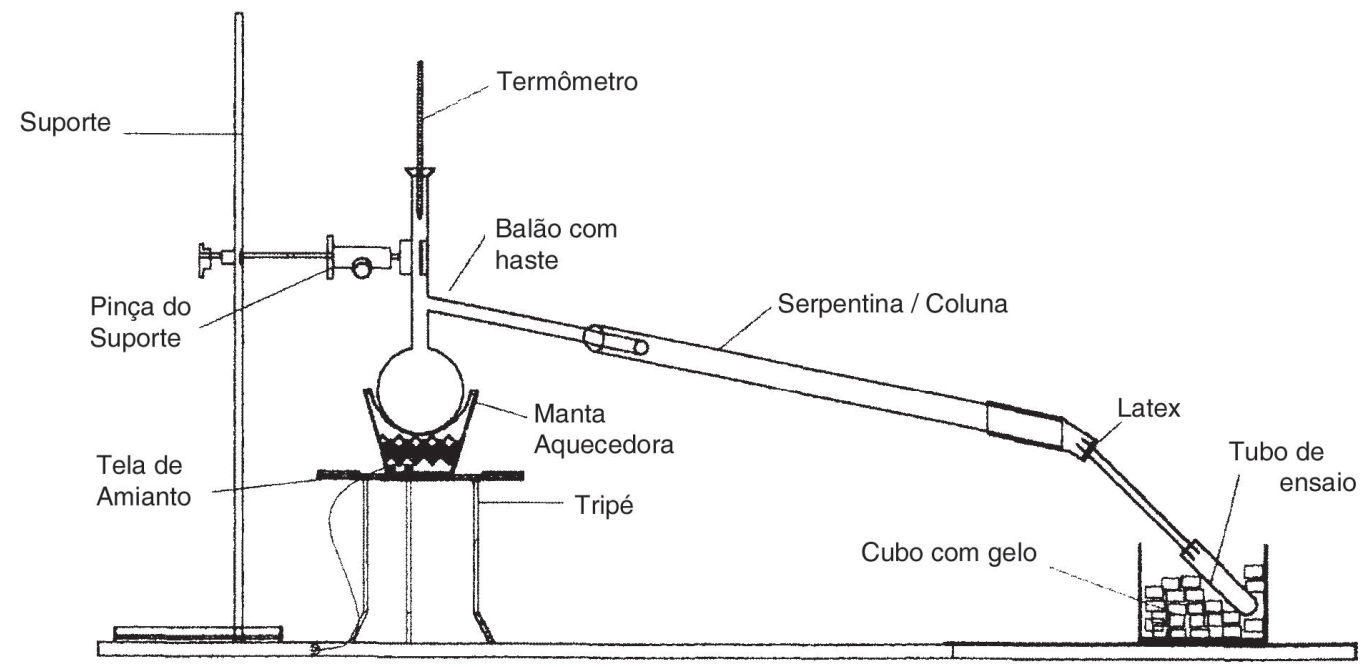

Figura 1. Esquema do procedimento utilizado na destilação fracionada do petróleo árabe leve.

\section{Resultados e Discussão.}

Um total de 43 alunos do Ensino Médio de uma escola pública de Londrina participaram neste estudo, onde o tema "O petróleo e sua destilação: uma abordagem experimental no Ensino Médio utilizando mapas conceituais", foi apresentado como extensão de uma unidade contínua em Química Orgânica. O trabalho teve a duração de 2 aulas de 50 minutos, por turma, e foi realizado com 21 alunos da $1^{\mathrm{a}}$ série e 22 alunos da $3^{\mathrm{a}}$ série. Cada turma foi organizada em 5 grupos de quatro e cinco alunos, de forma espontânea, com o objetivo de motiválos para uma atitude mais participativa. No trabalho foi usado um texto de abertura, como organizador prévio, elaborado com alguns conceitos de abordagem da Tecnologia, Ciência e Sociedade e um questionário. Posteriormente, desenvolveu-se um experimento que consistia na destilação fracionada de petróleo árabe leve. Os resultados 
foram monitorados, na primeira etapa, por um questionário e a construção de um mapa conceitual antes da atividade experimental. O questionário consistiu de duas perguntas dissertativas e três de múltipla escolha, para investigar o trabalho do referido texto. Durante a destilação fracionada do petróleo, promoveu-se um debate, onde alguns conceitos pertinentes ao petróleo e ao experimento foram acrescentados e outros esclarecidos. Esta etapa conduziu-se dentro de um contexto de teoria e laboratório, com uma abordagem do cotidiano dos alunos. Na segunda etapa, ao término da unidade de ensino de alguns conceitos de Química Orgânica, as equipes elaboraram um segundo mapa conceitual, objetivando-se coletar dados do conhecimento adquirido pelos alunos após a prática do laboratório. Durante a elaboração dos mapas conceituais, nos períodos pré e pós-atividade, não houve a participação da professora, para não interferir na investigação da estratégia de ensino utilizada neste trabalho. Nenhum dos alunos que participaram deste trabalho conhecia ou havia construído um mapa conceitual antes. Com os alunos da $1^{\mathrm{a}}$ série, verificou-se um resultado de $50 \%$ de aproveitamento do trabalho com o texto e, com os da $3^{a}$ série, o aproveitamento foi de $80 \%$. Nos primeiros mapas conceituais, elaborados pelos alunos da $1^{\mathrm{a}}$ série (Figura 2), os grupos colocaram a palavra "petróleo" como conceito mais geral, sendo que apenas um grupo relacionou com “energia”. Já nos segundos mapas conceituais, como mostra a mesma figura 3 , os grupos adotaram "energia" como conceito mais geral, significando que houve uma ampliação da estrutura do conhecimento.

Nos primeiros mapas conceituais elaborados houve algumas relações entre conceitos inadequados. Também não foram apresentadas hierarquias válidas para o conceito de craqueamento em 3 mapas. Embora com o trabalho do referido texto, em geral, estes apresentaram-se com diferenciação progressiva e reconciliação integradora, dos conceitos gerais aos específicos. Verificaram-se muitas falhas na formação de preposições e houve ausência de ligações transversais. Estas ligações abordam uma estrutura cognitiva muito positiva e foram verificadas 7 delas nos segundos mapas (Figura 2).
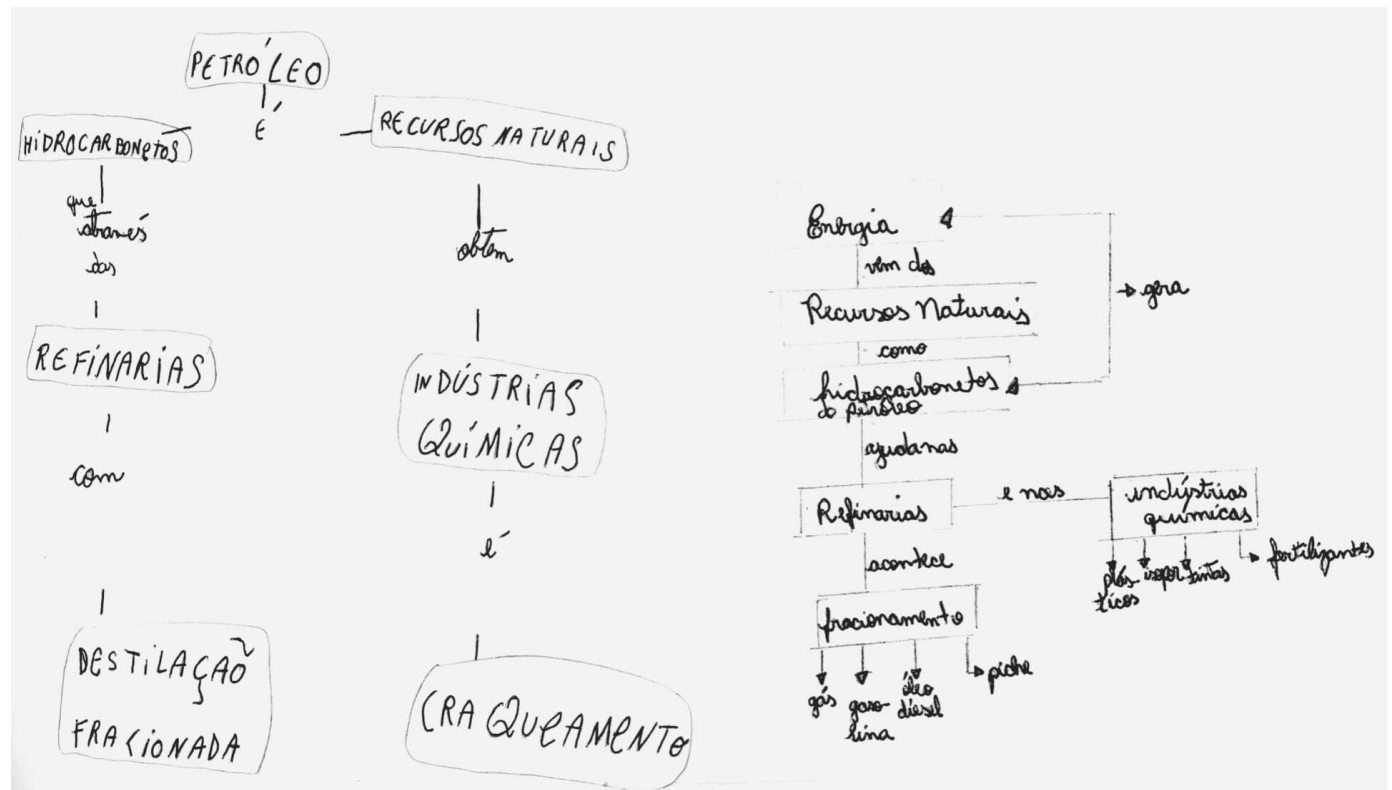

Figura 2. Mapa conceitual elaborado por um grupo da $1^{\mathrm{a}}$ série do Ensino Médio. À esquerda, mapa conceitual préatividade, e à direita, mapa conceitual pós-atividade. 
Dos cinco primeiros mapas conceituais elaborados pelos alunos da $3^{\mathrm{a}}$ série, três deles continham a palavra "petróleo" como conceito mais geral, e dois começaram com "recurso natural” (Figura 3). Também, três mapas apresentaram estrutura bidimensional, e dois unidimensional, contudo, estes últimos continham muitos exemplos. Apenas um dos mapas pós-atividade elaborados continha ligação transversal. Também, no segundo mapa construído, apenas 1 grupo apresentou uma não-hierarquia válida referente à destilação fracionada.

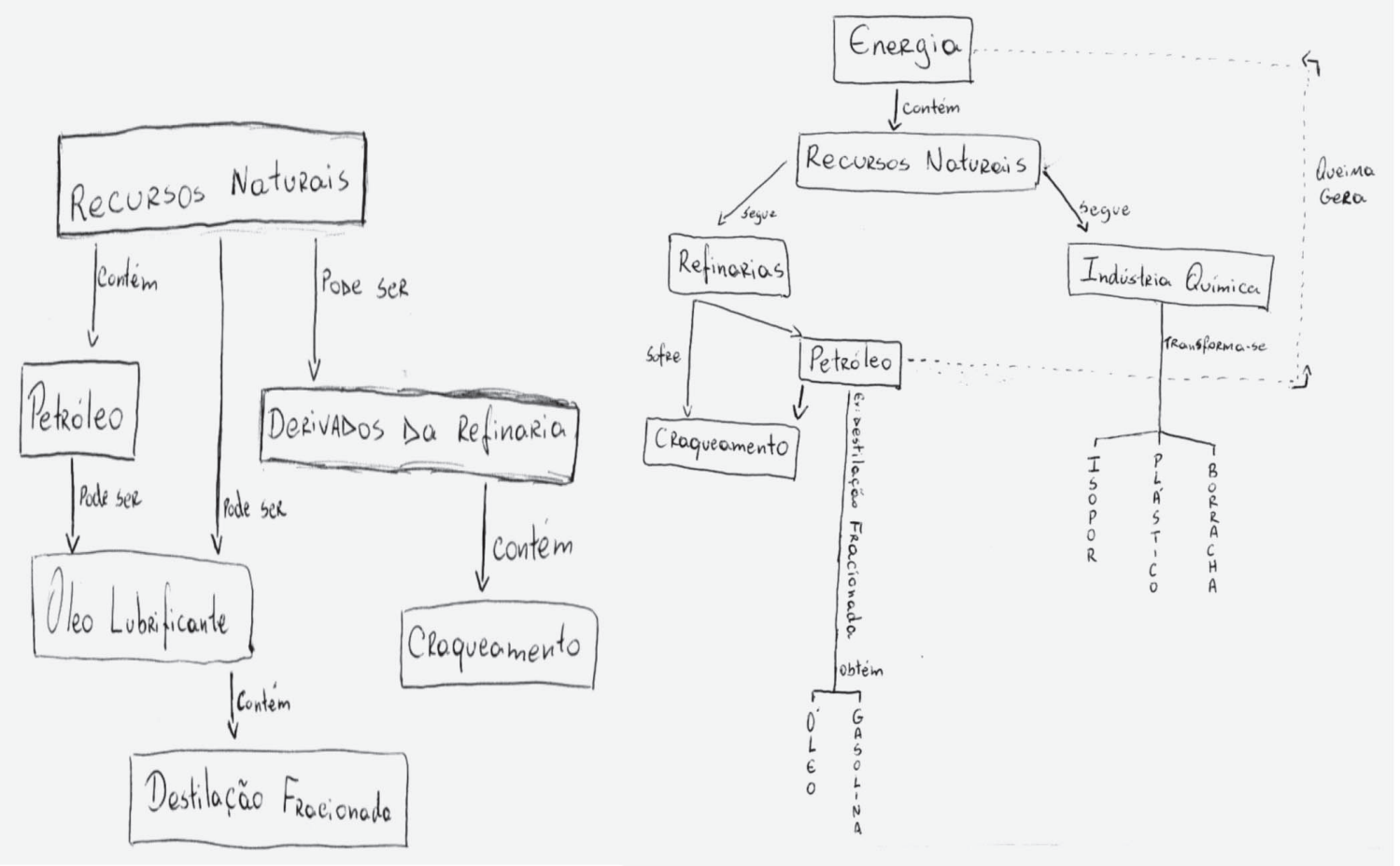

Figura 3. Mapa conceitual elaborado por um grupo da $3^{\mathrm{a}}$ série do Ensino Médio. À esquerda, mapa conceitual préatividade, e à direita, mapa conceitual pós-atividade.

Verificaram-se diferenças significativas entre o primeiro e o segundo mapa conceitual, notadamente naqueles construídos pelos alunos da $1^{\mathrm{a}}$ série.

\section{Conclusões}

Os conceitos químicos relacionados no laboratório podem tornar-se mais efetivos quando técnicas pedagógicas de ensino estiverem incorporadas.

Os professores podem utilizar mapas conceituais, para construção, reconstrução e inter-relações dos conceitos em prática de laboratório, em conjunto com um tema organizador apropriado para uma contextualização do cotidiano, com uma atribuição muito positiva da aprendizagem realmente significativa.

\section{Referências}

BENETASSO, D. L. ; ARAUJO, N. R. S. ; PEDRÃO, F. M. ; BUENO, E. A. S; ALMEIDA, F. A. S; BORSATO, D. O petróleo e sua destilação: uma abordagem experimental no ensino médio utilizando mapas conceituais. In: REUNIÃO ANUAL DA SOCIEDADE BRASILEIRA DE QUÍMICA, 25., 2002, Poços de Caldas. Anais... Poços de Caldas: SBQ, 2002.v.1.p.ED71-ED71. 
HERON, J. D.; NURRENBERN, S. C. Chemical Education Research: Improving Chemistry Learning. Journal of chemical Education, Easton, v.76, p.1354-1361, 1999.

MENEZES, L. C. Parâmetros Curriculares Nacionais (Ensino Médio). Parte III: Ciências da Natureza, Matemática e suas Tecnologias.Brasília, 2001.

MOREIRA, M. A.; NOVAK, J. D. Investigatíon em ensenãnza de lãs ciências em la Universidade de Cornell: esquemas teóricos, cuestiones centrales y abordes metodológicos. Enseñanza de Lãs Ciências, Barcelona, v.6, n.1, p.3-18, 1988.

NOVAK, J. D. , GOWIN, D. B. Aprender a aprender. Lisboa: Plátano Edições Técnicas, 1996. 